\title{
Time-resolved nonlinear ghost imaging: Route to hyperspectral single-pixel reconstruction of complex samples at $\mathrm{THz}$ frequencies
}

Olivieri, Luana, Totero Gongora, Juan, Pasquazi, Alessia, Peccianti, Marco

Luana Olivieri, Juan S. Totero Gongora, Alessia Pasquazi, Marco Peccianti, "Time-resolved nonlinear ghost imaging: Route to hyperspectral single-pixel reconstruction of complex samples at THz frequencies," Proc. SPIE 10902, Nonlinear Frequency Generation and Conversion: Materials and Devices XVIII, 1090219 (4 March 2019); doi: 10.1117/12.2508661

SPIE. Event: SPIE LASE, 2019, San Francisco, California, United States 


\title{
Time-resolved nonlinear ghost imaging: Route to hyperspectral single-pixel reconstruction of complex samples at $\mathrm{THz}$ frequencies
}

\author{
Luana Olivieri, Juan S. Totero Gongora*, Alessia Pasquazi and Marco Peccianti \\ Emergent Photonics Lab (EPic), Dept. of Physics and Astronomy, \\ University of Sussex, Brighton, BN1 9QH U.K.
}

\begin{abstract}
Terahertz (THz) is an innovative form of electromagnetic radiation providing unique spectroscopy capabilities in critical fields, ranging from biology to material science and security. The limited availability of high-resolution imaging devices, however, constitutes a major limitation in this field. In this work, we tackle this challenge by proposing an innovative type of time-space nonlinear Ghost-Imaging (GI) methodology that conceptually outperforms established single-pixel imaging protocols. Our methodology combines nonlinear pattern generation with time-resolved single-pixel measurements, as enabled by the state-of-the-art Time-Domain Spectroscopy (TDS) technique. This approach is potentially applicable to any wave-domain in which the field is a measurable quantity. The full knowledge of the temporal evolution of the transmitted field enables devising a new form of full-wave reconstruction process. This gives access not only to the morphological features of the sample with deeply subwavelength resolution but also to its local spectrum (hyperspectral imaging). As a target application, we consider hyperspectral $\mathrm{THz}$ imaging of a disordered inhomogeneous sample.
\end{abstract}

Keywords: terahertz, ghost imaging, nonlinear imaging, terahertz hyperspectral imaging, time-resolved ghost imaging, subwavelength hyperspectral imaging, time-domain spectroscopy, complex imaging, image reconstruction, phase-sensitive imaging.

\section{INTRODUCTION}

The reconstruction of amplitude and phase of complex field distributions is a standard issue in many domains, as in photonics ${ }^{1}$. Achieving full-wave characterisation is highly desirable in many applications, spanning from biological imaging to material characterisation, telecommunications, and optical time reversal ${ }^{2,3}$. Interestingly, this type of measurements is accessible in the terahertz (THz) frequency range through the time-domain spectroscopy (TDS) technique ${ }^{4}$. Several materials possess distinctive spectral signatures in the $\mathrm{THz}$ band, leading to the development of highly-sensitive spectroscopy applications, focusing on material discrimination and characterisation ${ }^{5-9}$. On the other hand, the realisation of field-sensitive (amplitude and optical delay) imaging devices still represents an open challenge. To date, thermal cameras are routinely used to characterise $\mathrm{THz}$ field distributions. These devices employ arrays of pyroelectric or bolometric sensors (based on different technologies) to detect the local average intensity of the signal.

To gain access to the field evolution, researchers have recently contemplated a range of alternative approaches, stemming from the concepts of Hartmann sensors and single-pixel ghost imaging (GI) protocols. While the former requires complex experimental configurations and fabrication, the simplicity of the latter approach is attracting an increasing amount of interest. In GI's original definition, the sample is illuminated with a predetermined sequence of optical patterns, and a bucket detector collects the resulting scattered light ${ }^{10,11}$. The correlation between the incident patterns and the transmitted field is then employed to reconstruct the target morphology. While in previous implementations researchers employed random arrangements of speckles as pattern sources, most recent applications exploit deterministic pattern distributions (an approach known as computational ghost imaging) ${ }^{12-14}$, removing the need of measuring the pattern distribution.

In an attempt to leverage the intrinsic capabilities of $\mathrm{THz}$ light, the combination of the GI scheme with field-sensitive

*Corresponding author email: j.totero-gongora@sussex.ac.uk 
TDS acquisition ${ }^{15}$ has been recently suggested. Such an approach, if achievable, would lead to the definition of a new type of time-resolved, field-based GI protocol where the full-temporal information can directly contribute to the image reconstruction process. In this context, however, one of the major practical challenges lies in the realisation of controllable structured beams. Spatial light modulators (SLM) that are routinely accessible at optical frequencies cannot be easily extended to the $\mathrm{THz}$ domain, mostly due to technological constraints. As such, a large part of recent research in this area has focused on the implementation of indirect patterning schemes (essentially masking devices), such as metallic masks or thin photoexcited silicon substrates ${ }^{16}$. Although these approaches have led to remarkable preliminary implementations of single-pixel THz imaging, they still possess several conceptual and practical drawbacks, in particular when considering the amplitude and phase characteristics of the THz pattern. In essence, those approaches neglect the spatiotemporal coupling in time-domain illumination, which is not consistent with the way the field information is spread in the space-time domain when an object is illuminated.

In this contribution, we propose a new approach to time-resolved, coherent single pixel imaging which outperforms standard GI implementations in relevant THz-imaging scenarios ${ }^{17}$. Our methodology, which has implications in several branches of wave physics, relies on the full-wave acquisition and direct generation of $\mathrm{THz}$ patterns through nonlinear conversion of an optical pulse. Our approach enables to reconstruct, without ambiguities, the full electromagnetic response of the sample (both in terms of amplitude and phase). As a result, nonlinear GI is a new and comprehensive tool for hyperspectral imaging that allows retrieving not only the sample's spatial features (with deeply subwavelength resolution) but also its local spectral properties.

\section{TIME-RESOLVED NONLINEAR GHOST IMAGING}

Our imaging protocol is inspired by the integration of GI with TDS acquisition. Unlike the standard GI, our methodology correlates the spatial information of the THz patterns with time-resolved single-pixel detection of the scattered field.

a
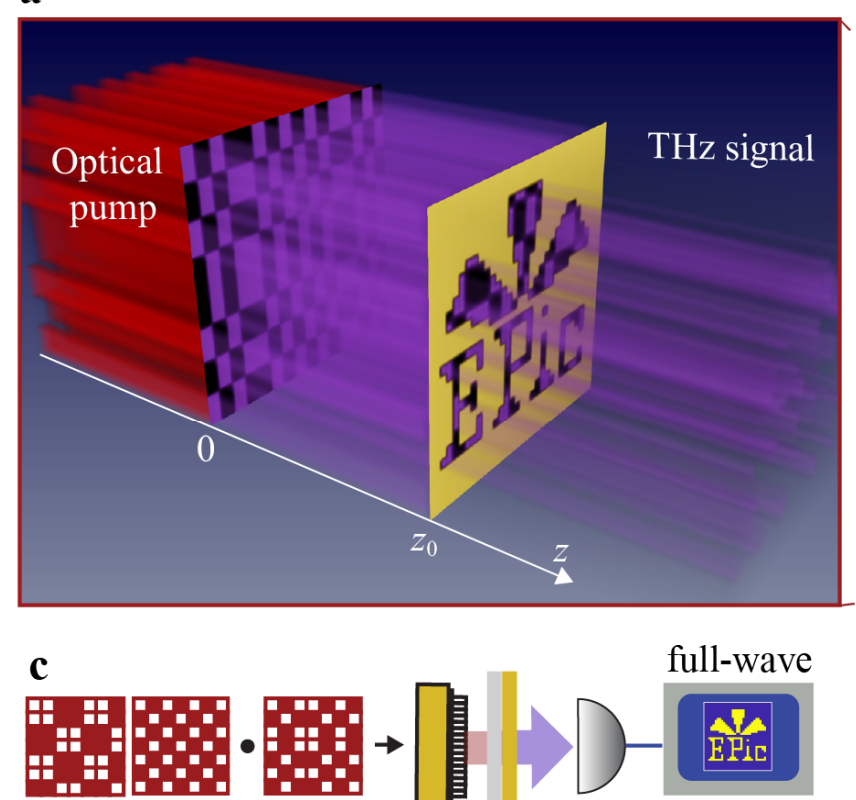

Deterministic patterns b

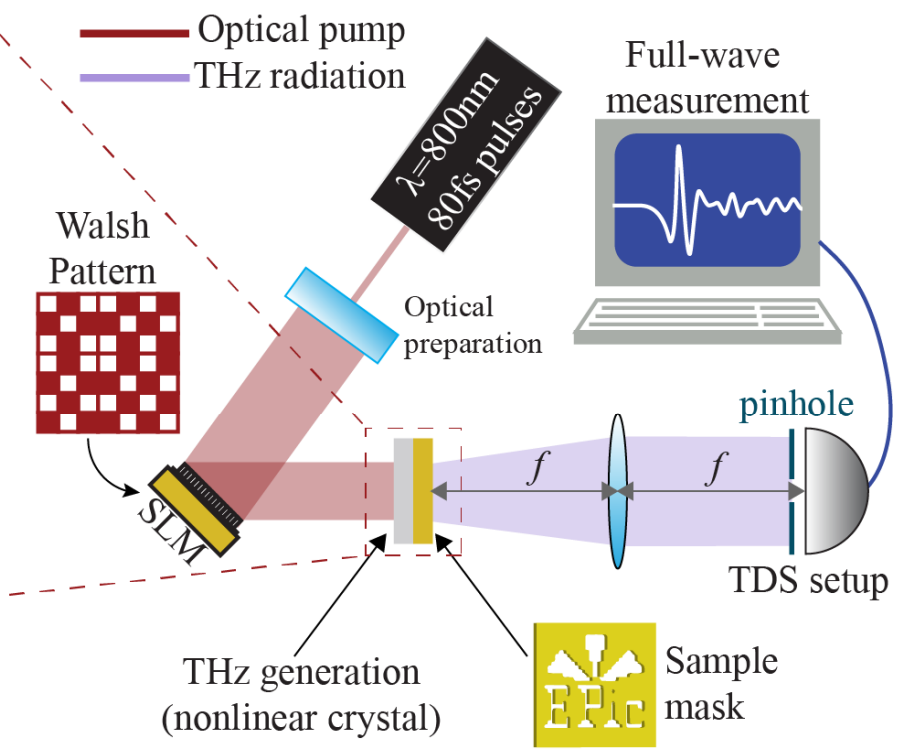

Figure 1: Time-resolved nonlinear ghost imaging. (a) Nonlinear generation and propagation of a THz pattern as it interacts with the sample. In this simulation, the emitted signal has a central wavelength of $\lambda=234 \mu m$, while the pixel size is $10 x 10 \lambda$. The nonlinear crystal thickness is $z_{0}=117 \mu m$. (b) Conceptual description of the imaging setup, illustrating the generation of THz light (purple beam) through nonlinear conversion of an optical pattern (red beam). (c) Schematic representation of the reconstruction using a Walsh-Hadamard encoding scheme. 
The reference nonlinear GI embodiment is shown in Figure 1. An optical pattern (red) is generated through a standard SLM device operating at optical frequencies (Fig 1b). Upon impinging on the surface of a generating crystal slab of thickness $z_{0}$ (e.g., $\mathrm{ZnTe}$ ), the optical pattern is nonlinearly converted to $\mathrm{THz}$ frequencies (purple). The THz pulse propagates across the crystal and interacts with the sample (Fig. 1a). It is worth noting that (compared to the state-of-the-art) we do not assume, at this stage, negligible propagation distance between object and sources, as at sub-wavelength scales not approximation can be effectively done in this framework. After the interaction, the transmitted $\mathrm{THz}$ field is collected through a single-pixel TDS detection setup. We implemented an imaging protocol based on the Walsh-Hadamard encoding scheme, which is known to maximise the Signal to Noise ratio (SNR) ${ }^{18}$. The Walsh patterns form an orthogonal set of binary matrices, which can be employed to expand any two-dimensional image in a deterministic fashion ${ }^{19}$.

The reconstructed image $S(x, y, t)$ is completely resolved in time and it is composed of amplitude and time information. It is defined as a projection over the optical pattern base $H_{n}(x, y)$ as follows:

$$
S(x, y, t)=\left\langle C_{n}(t) H_{n}(x, y)\right\rangle_{n}-\left\langle C_{n}(t)\right\rangle_{n}\left\langle H_{n}(x, y)\right\rangle_{n}
$$

where $\langle\cdots\rangle_{n}$ represents the expected value over the pattern ensemble (average over all the patterns) and $C_{n}(t)$ are expansion coefficients defined as:

$$
C_{n}(t)=\int \mathrm{d} x \mathrm{~d} y T(x, y, t) * E_{n}^{-}(x, y, t),
$$

* being the convolution operator. In Equations (1-2), $T(x, y, t)$ is the transmission function of the object and $E_{n}^{-}(x, y, t)$ are the THz patterned beams distribution immediately before the sample (which differs with the pattern at the source). In analogy with the standard GI implementations, the coefficients $C_{n}(t)$ coincide with the integrated scattered field as measured by the single-pixel detector. Differently from the optical case, however, our THz measurement is related to the full-wave electric field and not to the transmitted intensity.

Intuitively, the imaging protocol outlined in Eqs. (1-2) holds rigorously only if $E_{n}^{-}(x, y, t)$ preserves the same spatial information of $H_{n}(x, y)$. This constitutes a critical issue in THz single-pixel imaging. Generally speaking, in fact, several reasons exist for the initial pattern to modify in propagation, such as diffraction, dispersion or scattering. In particular, when considering subwavelength patterns (where the pixel size is smaller than the beam's central wavelength), the propagating wave naturally experiences spatiotemporal coupling, which can be detrimental as it can drastically change the structure of the pattern distribution ${ }^{20}$. While this effect could be mitigated, for example, by reducing the crystal thickness ${ }^{21}$, such procedure poses significant practical challenges ${ }^{22}$. Regardless, our results suggest that, especially when targeting very high resolutions or complex material responses, an ultimate limit always exists where the effects induced by spatiotemporal coupling cannot be no longer neglected.

Even in the presence of spatiotemporal coupling, however, the properties of the object remain encoded in the spatiotemporal domain. Those features can be accessed by deconvolving the data through an optimised propagation operator which eliminates any spatiotemporal coupling occurring within the nonlinear crystal. Our nonlinear approach related the $\mathrm{THz}$ source distribution with the optical excitation. This is different compared to approaches based on masking devices: in the latter, the pattern reaching the object's surface is defined as the convolution between the THz beam and the Green's function of the subwavelength masks, which is intrinsically different for each mask.

Following standard approaches, we define our inverse-propagation operator in the Fourier domain as follows:

$$
\boldsymbol{W}\left(k_{x}, k_{y}, k_{z}, \omega\right)=\frac{\boldsymbol{G}\left(k_{x}, k_{y}, k_{z}, \omega\right)^{*}}{\left|\boldsymbol{G}\left(k_{x}, k_{y}, k_{z}, \omega\right)\right|^{2}+\alpha N S R\left(k_{x}, k_{y}, k_{z}, \omega\right)},
$$


where $\boldsymbol{G}\left(k_{x}, k_{y}, k_{z}, \omega\right)$ is the dyadic Green's function in the Fourier domain, NSR $=1 / \mathrm{SNR}$ is the average spectral noise-to-signal ratio, and $\alpha$ is a fitting parameter ( $\alpha=0.1$ is our simulations). The action of the operator $\mathbf{W}$ is to inverse-propagate the experimental TDS trace through the crystal to eliminate the effects of spatiotemporal coupling. This allows us to retrieve the actual transmission function of the object in $T(x, y, t)$. In the absence of noise, the operator $\mathbf{W}$ coincides straightforwardly with the inverse Green's tensor. In the presence of experimental noise, vice-versa, assessment of the NSR term at the denominator is a strict requirement, as the transfer function cannot be univocally reconstructed using the Green's function ${ }^{23}$.

We verified the validity of our approach by simulating Eqs. (1-Error! Reference source not found.Error! Reference source not found.) to reconstruct a two-dimensional gold mask with deeply subwavelength features (as shown in Fig.2) ${ }^{17}$. The system was probed through a full set of Walsh pattern (Hadamard order $\mathrm{N}=32$ ), with a pixel size of $20 \mu \mathrm{m}$ and $\lambda=234$, propagating across a crystal of thickness $z_{0}=117 \mu \mathrm{m}$. To address the robustness of our procedure to experimental noise, we also included a white-noise term in the TDS trace with a statistical signal to noise ratio (SNR) of 5\% and compatible with state-of-the-art experimental conditions. We quantitatively assessed the fidelity of our approach, by implementing a Structural-Similarity-Index Method (SSIM) analysis. SSIM was recently proposed as an extension to the well-known Pearson correlation coefficient analysis and it provides a detailed comparison in terms of luminosity (mean value), contrast (variance) and structure (co-variance) of different spatial distributions. In the present case, two matrices are highly correlated when the SSIM value approaches 1, while they are uncorrelated for values below 0.14. In Fig. 2(c-d), we report the reconstructed images as obtained through a standard, fixed-time reconstruction (panel c) and through our approach (panel d). As can be evinced by the figure, in the fixed-time case the object morphology is hardly reconstructed even at the specific time corresponding to the maximum correlation (SSIM 31\%). Such incapability is a direct consequence of spatiotemporal coupling. The inverse propagated image, on the contrary, fully achieve the reconstruction of the transmission function $T(x, y, t)$ with deeply subwavelength features, as confirmed by the SSIM value $=84 \%$. It is worth mentioning that such value is rather close to the one obtained in the absence of "simulated" noise (88\%), suggesting that our methodology is potentially highly robust against experimental noise.

a
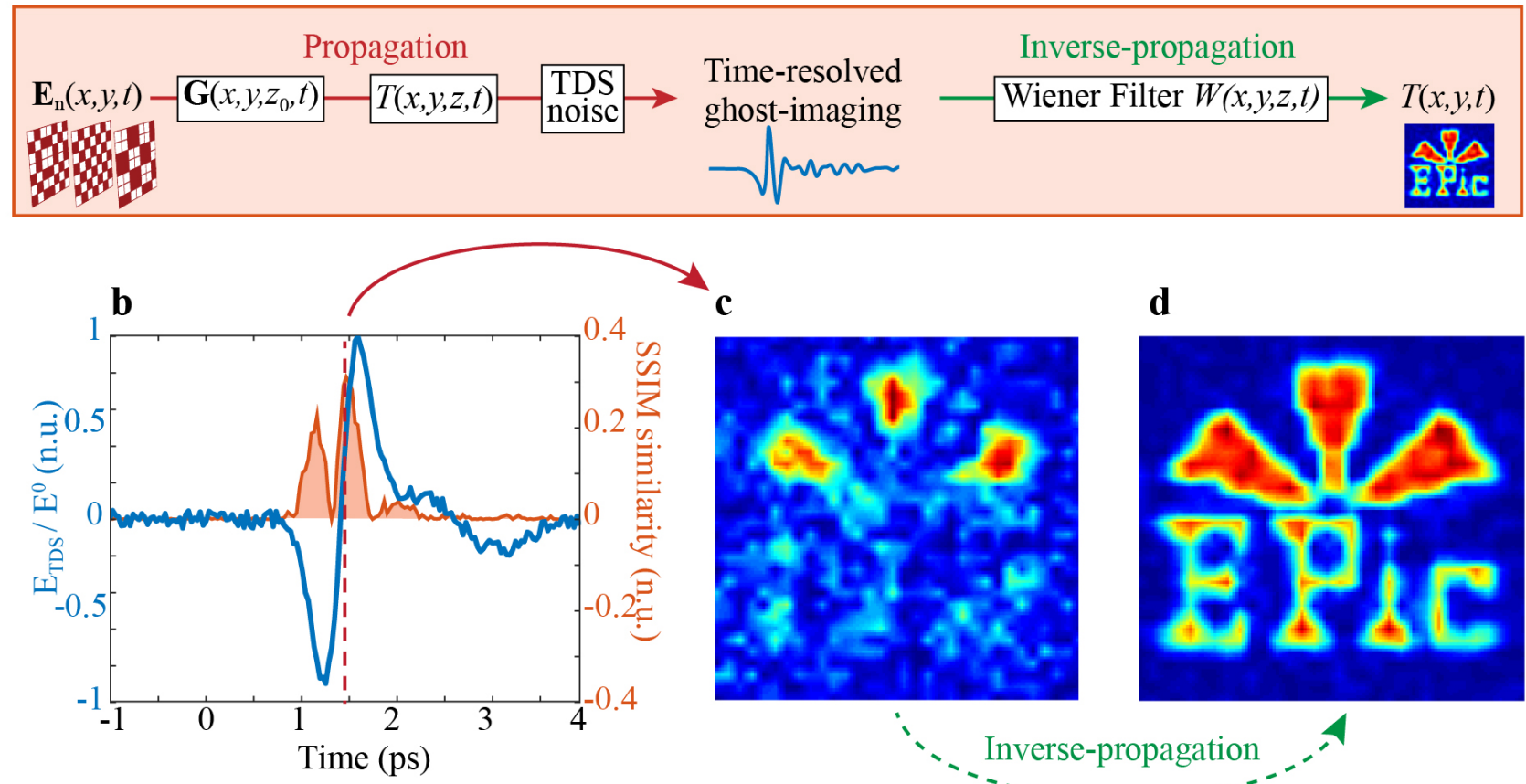

c

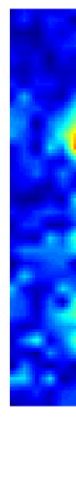

d

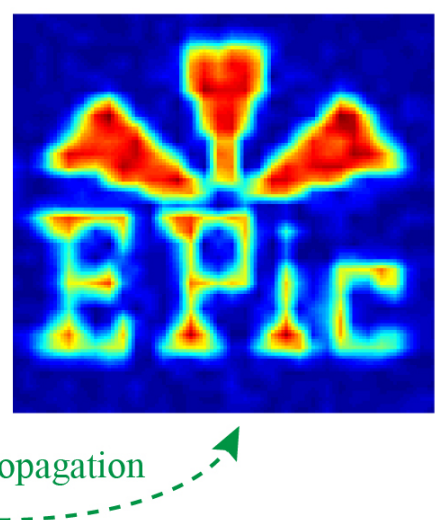

Figure 2: Time-resolved image reconstruction and inverse-propagation. (a) Scheme of the methodology used to reconstruct the sample's local transmission function with deeply sub-wavelength resolution. (b) Temporal evolution of the image TDS and SSIM correlation index. (c) Best reconstructed image reading the field distribution at the time corresponding to the maximum SSIM without Inverse propagation. Note that the best SSIM is not obtained in correspondence of the input THz field peak. (d) Image obtained applying the inverse-propagation operator to the spatiotemporal reconstructed image. 


\section{HYPERSPECTRAL IMAGING AND LOCAL SPECTRUM RETRIEVAL}

As a direct application of the nonlinear GI, we hereby show how this methodology brings significant advantages when considering spectral analysis. One of the most acknowledged features of TDS is the ability to retrieve the spectral response of complex samples accurately ${ }^{7,15}$. An intriguing question is whether single-pixel reconstruction, as enabled by our time-resolved nonlinear approach, could be employed to perform hyperspectral imaging, i.e. to access the local spectral information for each point of the reconstructed image. Such a measurement would be undoubtedly pivotal in the development of new approaches to the spectral analysis and material recognition of complex semi-transparent samples. We considered an ideal semi-transparent target composed of a random droplet distribution of two different material species, each characterised by rather different spectral profiles (Fig. 3). In particular, we considered two gaussian transmission spectra with the same waist but centred at different frequencies $(1.8 \mathrm{THz}$ and $1 \mathrm{THz}$ for the first and second species, respectively). We illuminated the target through a broadband $\mathrm{THz}$ source centred at $240 \mu \mathrm{m}(1.25 \mathrm{THz})$ that propagates through a $117 \mu \mathrm{m}$ thick nonlinear crystal. Spatially, we considered Hadamard patterns composed of $64 \times 64$ pixels, a pixel size corresponding to $2 \lambda(500 \mu \mathrm{m})$. As illustrated in Fig 3, our methodology is capable to retrieve the full-wave transmission function $T(x, y, \omega)$ of the sample accurately, providing us with a detailed description of the sample both in space and frequency. Remarkably, the spectra in each point of the reconstructed image closely resembles the material spectra (Fig. 3c,f) as expected in an hyperspectral imaging condition. Moreover, the two species can be easily discriminated by retrieving the spatial distribution corresponding to the different peaks of the local spectrum, as depicted in Fig 3c-d and 3f-g.

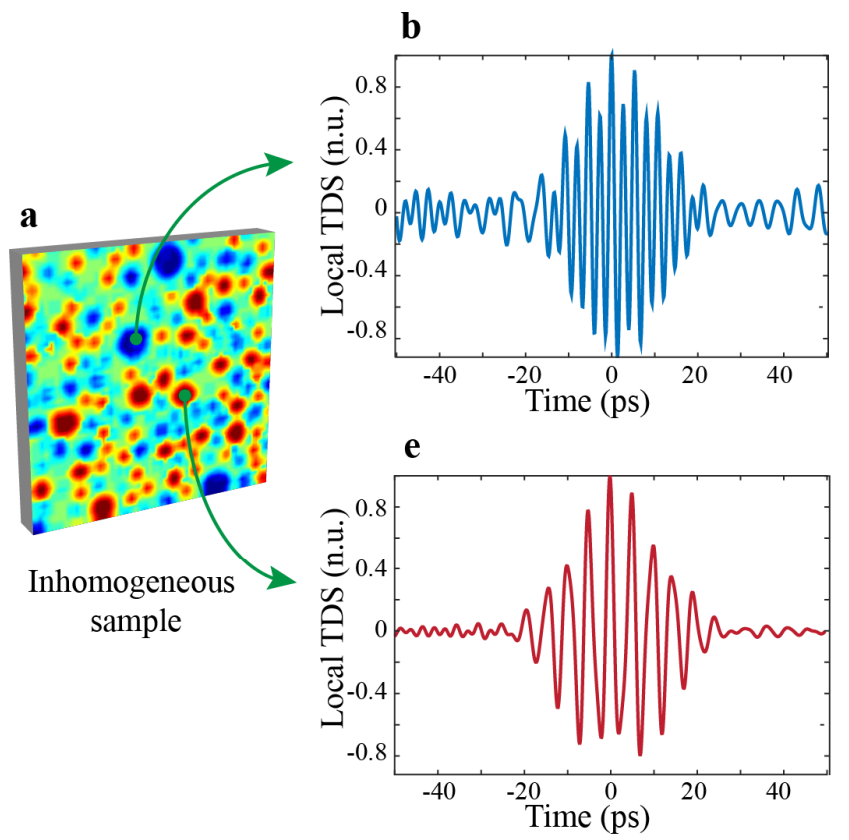

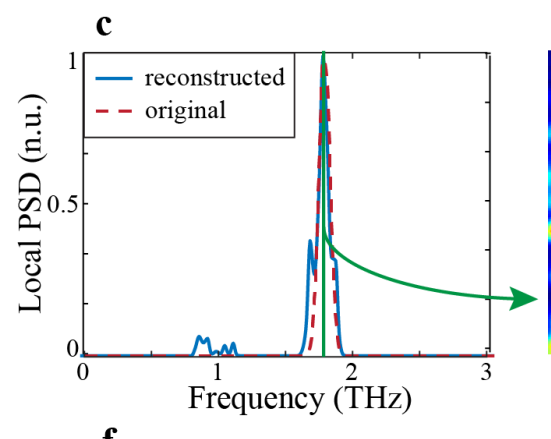

d

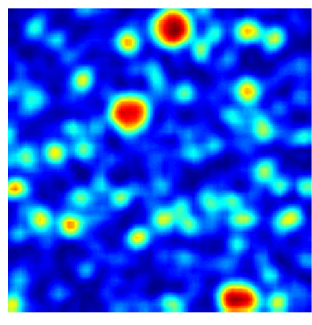

First species

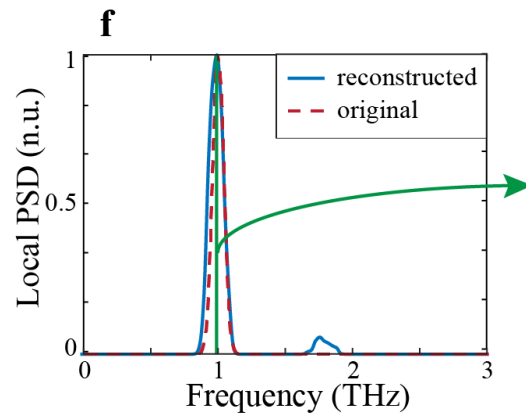

g

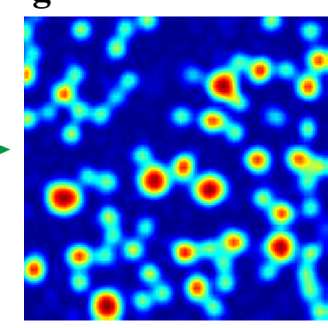

Second species

Figure 3: Single-pixel hyperspectral imaging using time-resolved nonlinear GI. (a) Complex inhomogeneous sample composed of a random distribution of two different material species (red and blue droplets). (b-c) Local temporal TDS trace (b) and corresponding PSD distribution (c) in a "first species" location (blue droplets). As illustrated in panel (c), the reconstructed spectrum (blue line) is remarkably correlated to the material one (dashed red line). (d) Spatial image reconstructed at the PSD peak, $v=1.8 \mathrm{THz}$, showing the first species spatial arrangement. (e-f-g) Same as (b-c-d) for the second species (red dots in (a)) and PSD peak at $v=1$ THz. 


\section{ACKNOWLEDGEMENTS}

We acknowledge the support of the U.K. Quantum Technology Hub for Sensors and Metrology, EPSRC, under Grant EP/M013294/1. This research received funding from the European Union's "Horizon 2020" research and innovation programme, grant agreement no 725046 .

\section{REFERENCES}

[1] Rivenson, Y., Zhang, Y., Günaydın, H., Teng, D. and Ozcan, A., "Phase recovery and holographic image reconstruction using deep learning in neural networks," Light: Science \& Applications 7(2), 17141 (2018).

[2] Zhang, X. C., Shkurinov, A. and Zhang, Y., "Extreme terahertz science," Nature Photonics 11(1), 16-18 (2017).

[3] Papadopoulos, I. N., Jouhanneau, J.-S., Poulet, J. F. A. and Judkewitz, B., "Scattering compensation by focus scanning holographic aberration probing (F-SHARP)," Nature Photonics 11(2), 116-123 (2017).

[4] Mittleman, D. M., "Twenty years of terahertz imaging [Invited]," Optics Express 26(8), 9417 (2018).

[5] Saeedkia, D., [Handbook of Terahertz Technology for Imaging, Sensing and Communications], Elsevier (2013).

[6] Dhillon, S. S., Vitiello, M. S., Linfield, E. H., Davies, A. G., Hoffmann, M. C., Booske, J., Paoloni, C., Gensch, M., Weightman, P., Williams, G. P., Castro-Camus, E., Cumming, D. R. S., Simoens, F., Escorcia-Carranza, I., Grant, J., Lucyszyn, S., Kuwata-Gonokami, M., Konishi, K., Koch, M., et al., "The 2017 terahertz science and technology roadmap," Journal of Physics D: Applied Physics 50(4), 043001 (2017).

[7] Dexheimer, S. L., [Terahertz spectroscopy: principles and applications], CRC press (2007).

[8] Pickwell, E. and Wallace, V. P., "Biomedical applications of terahertz technology,” Journal of Physics D: Applied Physics 39(17), 301-310 (2006).

[9] Zeitler, J. A., Taday, P. F., Newnham, D. a, Pepper, M., Gordon, K. C. and Rades, T., "Terahertz pulsed spectroscopy and imaging in the pharmaceutical setting--a review.," The Journal of pharmacy and pharmacology 59(2), 209-223 (2007).

[10] Shapiro, J. H. and Boyd, R. W., "The physics of ghost imaging," Quantum Information Processing 11(4), 949-993 (2012).

[11] Padgett, M. J. and Boyd, R. W., “An introduction to ghost imaging: quantum and classical,” Phil. Trans. R. Soc. A 375(2099), 20160233 (2017).

[12] Gatti, A., Brambilla, E., Bache, M. and Lugiato, L. A., "Correlated imaging, quantum and classical," Physical Review A - Atomic, Molecular, and Optical Physics 70(1), 013802-013802 (2004).

[13] Bache, M., Brambilla, E., Gatti, a and Lugiato, L., "Ghost imaging schemes: fast and broadband.," Optics Express 12(24), 6067-6081 (2004).

[14] Ferri, F., Magatti, D., Lugiato, L. A. and Gatti, A., "Differential ghost imaging," Physical Review Letters 104(25), 253603-253603 (2010).

[15] Stantchev, R. I., Mansfield, J. C., Edginton, R. S., Hobson, P., Palombo, F. and Hendry, E., "Subwavelength hyperspectral THz studies of articular cartilage," Scientific Reports 8(1), 6924 (2018).

[16] Stantchev, R. I., Sun, B., Hornett, S. M., Hobson, P. A., Gibson, G. M., Padgett, M. J. and Hendry, E., "Noninvasive, near-field terahertz imaging of hidden objects using a single-pixel detector," Science Advances 2(6), e1600190 (2016).

[17] Olivieri, L., Totero Gongora, J. S., Pasquazi, A. and Peccianti, M., "Time-Resolved Nonlinear Ghost Imaging," ACS Photonics 5(8), 3379-3388 (2018).

[18] Davis, D. S., "Multiplexed imaging by means of optically generated Kronecker products: 1. The basic concept," Applied Optics 34(7), 1170-1176 (1995).

[19] Eldar, Y. C. and Kutyniok, G., eds., Compressed sensing: theory and applications, Cambridge University Press, Cambridge ; New York (2012). 
[20] Peccianti, M., Clerici, M., Pasquazi, A., Caspani, L., Ho, S. P., Buccheri, F., Ali, J., Busacca, A., Ozaki, T. and Morandotti, R., "Exact Reconstruction of THz Sub- $\lambda$ Source Features in Knife-Edge Measurements," IEEE Journal of Selected Topics in Quantum Electronics 19(1), 8401211-8401211 (2013).

[21] Walsh, D. A., Cliffe, M. J., Pan, R., Snedden, E. W., Graham, D. M., Gillespie, W. A. and Jamison, S. P., "Role of misalignment-induced angular chirp in the electro-optic detection of THz waves," Opt. Express, OE 22(10), 12028-12037 (2014).

[22] Peters, L., Tunesi, J., Pasquazi, A. and Peccianti, M., "High-energy terahertz surface optical rectification," Nano Energy 46, 128-132 (2018).

[23] Khare, K., Fourier optics and computational imaging, Wiley, John Wiley \& Sons Ltd, Chichester, West Sussex (2016). 